\title{
SÁndor Jeneiఠ Correction to: The Hahn Embedding Theorem for a Class of Residuated Semigroups
}

\begin{abstract}
Let $\mathcal{O}$ be the class of odd involutive $\mathrm{FL}_{e}$-chains which admit finitely many idempotent elements. The price for describing the structure of such a rich class as $\mathcal{O}$ in [26] by such simple mathematical objects as linearly ordered abelian groups was that the related construction method, called partial lex product was quite complex. But in order to describe the structure of $\mathcal{O}$, even the notion of partial lex products is not sufficiently general. One more tweak is needed, a slightly even more complex construction, called partial sublex product, introduced here.
\end{abstract}

\section{Correction to: Studia Logica (2020) 108: 1161-1206 DOI 10.1007/s11225-019-09893-y}

\section{The error}

The main result of [26] (hereinafter the original statement) falsely states that every algebra in $\mathcal{O}$ can be constructed by applying finitely many times the partial lex product construction using totally ordered abelian groups. However, it holds true that (hereinafter the correct statement) every algebra in $\mathcal{O}$ can be constructed by applying finitely many times the partial sublex product construction using totally ordered abelian groups. In the sequel we explain why the original statement is false, we introduce the partial sublex product construction, and show what modifications are needed in [26] to obtain the correct statement. Knowledge of notions in [26] will be assumed.

\footnotetext{
The original article can be found online at https://doi.org/10.1007/ s11225-019-09893-y.

The original online version of this article was revised: The corresponding author name was incorrectly published as "Sándor A. Jenei", the correct name is "Sándor Jenei".
}

Presented by Daniele Mundici; Received February 18, 2020 
Why is it that the original statement cannot hold true? An insight can be gained from the synthesis of the following three observations.

1. Let $\mathbf{A}$ and $\mathbf{B}$ be totally ordered abelian groups and let $\mathbf{G}=\mathbf{A} \overrightarrow{\times} \mathbf{B}$ be their lexicographic product. We are interested in characterizing subgroups of $\mathbf{G}$. Clearly, if $\mathbf{A}_{1} \leq \mathbf{A}$ and $\mathbf{B}_{1} \leq \mathbf{B}$ then $\mathbf{A}_{1} \overrightarrow{\times} \mathbf{B}_{1} \leq \mathbf{G}$, but not all subgroups of $\mathbf{G}$ are of that form. For example, if $\mathbf{A}=\mathbf{B}$ then $\mathbf{G}$ has a subgroup over $\{(x, x): x \in A\}$. This subgroup lies 'diagonally' in the direct decomposition $\mathbf{A} \overrightarrow{\times} \mathbf{B}$ of $\mathbf{G}^{1}$, and Birkhoff's famous subdirect representation theorem is exactly about such diagonal situations, in general.

2. The universe of the group part of the partial lex products $\mathbf{A}=\mathbf{X}_{\mathbf{Z}_{\mathbf{V}}} \overrightarrow{\times} \mathbf{Y}$ and $\mathbf{B}=\mathbf{X}_{\mathbf{V}} \overrightarrow{\times} \mathbf{Y}$ is equal to $V \times Y_{g r}$, so it is never diagonal.

3. We shall shortly prove in Proposition B.2 that one can freely replace the group part of an odd involutive $\mathrm{FL}_{e}$-algebra by any other lattice-ordered abelian subgroup of it (e.g. by a diagonal one), thus obtaining another odd involutive $\mathrm{FL}_{e}$-algebra.

It follows that if we replace the group part of an odd involutive $\mathrm{FL}_{e}$-algebra by a diagonal subgroup of it, then we get an odd involutive $\mathrm{FL}_{e}$-algebra which cannot be constructed by the partial lex product construction. Since this procedure does not change the number of idempotent elements of the algebra, a more general construction is needed to describe the class $\mathcal{O}$.

Where is the error in the proof of the original statament? Formally, the argument in the last sentence of the proof of Lemma 6.2 is the invalid one. The proof of Lemma 6.2 cannot be fixed, since the very last statement of it, namely that $\mathbf{X}_{\mathbf{g}} \mathbf{r} \cong \beta\left(\mathbf{X}_{\mathbf{g r}}\right) \overrightarrow{\times} \mathbf{k e r}_{\beta}$, is false, in general: given a linearly ordered abelian group $G$ and a convex subgroup $C$ of it, $G$ is not necessarily isomorphic to $G / C \overrightarrow{\times} C$. It is shown by the fact that in general $G$ cannot even be embedded into $G / C \overrightarrow{\times} C$, see [25, Example 4.5.2]. Since Lemma 6.2 was used in the one-step decomposition lemmas (in Lemmas 9.4 and 10.2), and since these were applied in the proof of the main representation theorem (Theorem 11.1), the main representation theorem and its corollary, the generalization of Hahn's embedding theorem in Corollary 11.5, have to be changed slightly.

\footnotetext{
${ }^{1}$ We shall use this term to mean that the universe of the subalgebra is not the direct product of the universe of a subalgebra of $\mathbf{A}$ and the universe of a subalgebra of $\mathbf{B}$.
} 


\section{How to Fix the Error}

We present the necessary changes to be made in [26] to obtain the correct statement. The main contribution (the novelty) of the present corrigendum is the fixing of Lemma 6.2, which will be done in Lemma B.4. That aside, the modifications listed below are only to carry over the difference between Lemma 6.2 and Lemma B.4 throughout the introduction and the proof of the original statement.

- Change the word "lexicographic" to "sub-lexicographic" in the Introduction (two instances). Change the word "lexicographic" to "sublex" in the abstract (two instances), in the title of Section 4, in the section before Remark 4.5, in the sentence before Theorem 11.1, and in Remark 11.2.

- Add the following to the end of Definition 1.2:

The lexicographic product $\mathbf{A} \overrightarrow{\times} \mathbf{B}$ of two $\mathrm{FL}_{e^{-}}$chains $\mathbf{A}$ and $\mathbf{B}$ is an $\mathrm{FL}_{e^{-}}$ chain over the lexicographic product of their respective universes such that all operations are defined coordinatewise.

- Add the following to the end of Example 3.2:

Moreover, it turns out that in order to describe the class of odd involutive $\mathrm{FL}_{e}$-chains having finitely many idempotent elements in its full generality, it is also possible to take such subalgebras of the group-part of the constructed algebra which are not the direct products of their projections. It motivates the most general construction, called partial sublex products in Definition B.3.

- Replace the first sentence of the paragraph after Definition 4.1 by the following:

Next, we introduce a construction, called partial lexicographic product (or partial lex product, or partial lex extension) with four slightly different variations in Definition 4.2. This definition lays the foundation for the most general construction in Definition B.3, needed for our structural description purposes.

- Add the following after the proof of Theorem 4.4:

Definition B.1. We introduce the following notation. Let $\mathbf{A}_{1}, \mathbf{A}_{2}$ and $\mathbf{D}$ be $\mathrm{FL}_{e}$-algebras, and let $\mathbf{D} \leq \mathbf{A}_{1} \overrightarrow{\times} \mathbf{A}_{2}$. If $\nu$, the projection operation to the first coordinate maps $\mathbf{D}$ onto $A_{1}$, that is, if $\nu(D)=\left\{a_{1} \in A_{1}\right.$ : there $\left.\operatorname{exists}\left(a_{1}, a_{2}\right) \in D\right\}=A_{1}$ then we denote this by 


$$
\mathbf{D} \leq_{\nu} \mathbf{A}_{1} \overrightarrow{\times} \mathbf{A}_{2}
$$

If $\mathbf{D}$ is only embedded into $\mathbf{A}_{1} \overrightarrow{\times} \mathbf{A}_{2}$ such that for its image $\varphi(\mathbf{D})$ it holds true that $\varphi(\mathbf{D}) \leq_{\nu} \mathbf{A}_{1} \overrightarrow{\times} \mathbf{A}_{2}$, then we denote this by

$$
\mathbf{D} \hookrightarrow{ }_{\nu} \mathbf{A}_{1} \overrightarrow{\times} \mathbf{A}_{2} .
$$

Next, we introduce the construction for the key result of the paper, called partial sublex product (or partial sublex extension). Consider an odd involutive $\mathrm{FL}_{e}$-algebra $\left(X, \leq, \oplus, \rightarrow_{\circledast}, t, f\right)$. Recall that the invertible elements of $\mathbf{X}$ are in $X_{g r}$. Our plan is to take a subalgebra $\mathbf{H}$ of $\mathbf{X}_{\mathbf{g r}}$, and to consider the set

$$
Y=\left(X \backslash X_{g r}\right) \cup H
$$

Then:

Proposition B.2. $Y$ is a nonempty subuniverse of $X$.

Proof. $Y$ is closed under multiplication. Indeed, the product of $a \in Y$ by a non-invertible element $b \in X \backslash X_{g r}$ is non-invertible: if $c=a \oplus b$ were invertible then $t=c^{-1} \oplus c=c^{-1} \oplus(a \oplus b)=\left(c^{-1} \oplus a\right) \oplus b$ would hold and thus $c^{-1} \oplus a$ would be the inverse of $b$, contrary to assumption. Hence $Y \oplus\left(X \backslash X_{g r}\right) \subseteq X \backslash X_{g r}$. Therefore,

$$
\begin{aligned}
Y \otimes Y & =Y \otimes\left[\left(X \backslash X_{g r}\right) \cup H\right] \\
& =\left[Y \circledast\left(X \backslash X_{g r}\right)\right] \cup(Y \oplus H) \\
& \subseteq\left(X \backslash X_{g r}\right) \cup\left(\left[\left(X \backslash X_{g r}\right) \cup H\right] \oplus H\right) \\
& =\left(X \backslash X_{g r}\right) \cup\left(\left(X \backslash X_{g r}\right) \oplus H\right) \cup(H \oplus H), \\
& \subseteq\left(X \backslash X_{g r}\right) \cup\left(\left(X \backslash X_{g r}\right) \oplus Y\right) \cup(H \oplus H) \\
& \subseteq\left(X \backslash X_{g r}\right) \cup\left(X \backslash X_{g r}\right) \cup H \\
& =Y
\end{aligned}
$$

and we are done. $Y$ is clearly closed under the residual complement operation ' of $X$, and hence under $\rightarrow_{\circledast}$, too. Finally, $t \in Y$ since $t \in H \subseteq Y$, and hence $f=t^{\prime} \in Y$, too.

These considerations lead to:

Definition B.3. Adapt the notation of Definition 4.2. Let $\mathbf{A}=\mathbf{X}_{\mathbf{Z}_{\mathbf{V}}} \overrightarrow{\times} \mathbf{Y}$ and $\mathbf{B}=\mathbf{X}_{\mathbf{V}} \vec{\overrightarrow{ }} \mathbf{Y}$. Recall that $A=(V \times Y) \cup(Z \times\{\top\}) \cup(X \times\{\perp\})$, $B=(V \times Y) \cup(X \times\{\top\})$, and that the group part $\mathbf{A}_{\mathbf{g r}}$ of $\mathbf{A}$, as well as the group part $\mathbf{B}_{\mathrm{gr}}$ of $\mathbf{B}$ is the group $\mathbf{V} \overrightarrow{\times} \mathbf{Y}_{\mathrm{gr}}$ which is the subalgebra of $\mathbf{A}$ and of $\mathbf{B}$ over $V \times Y_{g r}$ in both cases. Let

$$
\mathbf{H} \leq_{\nu} \mathbf{V} \overrightarrow{\times} \mathbf{Y}_{\mathbf{g r}}
$$


Replace $\mathbf{A}_{\mathbf{g r}}$ in $\mathbf{A}$ and $\mathbf{B}_{\mathbf{g r}}$ in $\mathbf{B}$ by $\mathbf{H}$ to obtain $\mathbf{A}_{\mathbf{H}}$ and $\mathbf{B}_{\mathbf{H}}$, respectively. More formally, let

$$
\begin{aligned}
& A_{H}=H \cup\left(V \times\left(Y \backslash Y_{g r}\right) \cup(Z \times\{\top\}) \cup(X \times\{\perp\}),\right. \\
& B_{H}=H \cup\left(V \times\left(Y \backslash Y_{g r}\right)\right) \cup(X \times\{\top\}) .
\end{aligned}
$$

Then, from Proposition B.2 it follows that $A_{H}$ and $B_{H}$ are closed under all operations of $\mathbf{A}$ and $\mathbf{B}$ (including the residual complement operation), respectively, and thus $\mathbf{A}_{\mathbf{H}}$ is a subalgebra of $\mathbf{A}$ over $A_{H}$, and $\mathbf{B}_{\mathbf{H}}$ is a subalgebra of $\mathbf{B}$ over $B_{H}$ by Theorem 4.4. Therefore, $\mathbf{A}_{\mathbf{H}}$ and $\mathbf{B}_{\mathbf{H}}$ are odd involutive $\mathrm{FL}_{e}$-algebras. As explained in Section 1, these cannot be constructed by the partial lex product construction, in general.

It has been beneficial to introduce the partial lex product construction in its full generality for demonstrating the constructional power of it. However, an important particular instance is if $\mathbf{Y}$ is cancellative, and this is all that we need in the sequel. Then $Y \backslash Y_{g r}=\emptyset$ and hence $A_{H}$ and $B_{H}$ become simpler:

$$
A_{H}=H \cup(Z \times\{\top\}) \cup(X \times\{\perp\}), B_{H}=H \cup(X \times\{\top\}) .
$$

This way both definitions become independent of $V$ since we can also equivalently assume

$$
\mathbf{H} \leq \mathbf{Z} \overrightarrow{\times} \mathbf{Y}
$$

instead of (B.1), where in the type II case $\mathbf{Z}=\mathbf{X}_{\mathbf{g r}}$. For this case (when $\mathbf{Y}$ is cancellative) we are going to use the following notation: $\left.\mathbf{X}_{(\mathbf{Z} \times \mathbf{Y}}\right)_{\mathbf{H}}$ for $\mathbf{A}_{\mathbf{H}}$, and $\mathbf{X}_{\left(\mathbf{x}_{\mathbf{g r}} \times \mathbf{Y}\right)_{\mathbf{H}}}$ for $\mathbf{B}_{\mathbf{H}}$, and will call them the type I partial sublexicographic (shortly sublex) product of $\mathbf{X}, \mathbf{Z}, \mathbf{Y}$ and $\mathbf{H}$, and the type II partial sublex product of $\mathbf{X}, \mathbf{Y}$ and $\mathbf{H}$, respectively.

- In Section 5 in the "Sketch of the main theorem" part replace the two item 3's by the following, respectively.

3. Finally we will recover $\mathbf{X}$, up to isomorphism, as the type I partial sublex product of $\gamma(\beta(\mathbf{X})), \gamma\left(\beta\left(\mathbf{X}_{\tau \geq u}^{E}\right)\right), \overline{\mathbf{k e r}_{\beta}}$, and a subgroup $\mathbf{G}$ of $\gamma\left(\beta\left(\mathbf{X}_{\tau \geq u}^{E_{c}}\right)\right) \overrightarrow{\times} \overline{\mathbf{k e r}_{\beta}}$, in Section 9.

3. Finally we will recover $\mathbf{X}$, up to isomorphism, as the type II partial sublex product of $\mathbf{X}_{\tau \geq u}, \mathbf{X}_{\tau \geq u}^{T}, \operatorname{ker}_{\beta}$, and a subgroup $\mathbf{G}$ of $\mathbf{X}_{\tau \geq u}^{T_{c}} \overrightarrow{\times} \overline{\mathbf{k e r}_{\beta}}$, in Section 10. 
- In the first sentence of the subsequent paragraph replace "(either type III or IV in Sections 9 and 10, respectively)" by "(either type I or II in Sections 9 and 10, respectively)".

- Now comes the main contribution in correcting the error mentioned in Section 1. Insert the following to the beginning of Section 6 .

An abelian group $\mathbf{A}$ is called divisible if for any $g \in A$ and $n$ positive integer there exists $a \in A$ such that $\underbrace{a \oplus \ldots \oplus a}_{n}=g$. For any abelian group $\mathbf{A}$

there exists a minimal divisible abelian group $\overline{\mathbf{A}}$ containing $\mathbf{A} . \overline{\mathbf{A}}$ is uniquely determined up to isomorphism and called the divisible hull (or injective hull) of $\mathbf{A}$ ([24]). The proof of the next lemma (unlike the rest of the paper) is written in the usual additive notation of the theory of abelian groups. ${ }^{2}$

Lemma B.4. Let $\mathbf{G}$ be a linearly ordered abelian group (denote its group operation by + ) and $\mathbf{C}$ be a convex subgroup of $\mathbf{G}$. Then $\mathbf{G} \hookrightarrow_{\nu} \mathbf{G} / \mathbf{C} \overrightarrow{\times} \overline{\mathbf{C}}$.

Proof. Consider $\mathbf{L}=\langle\mathbf{G}, \overline{\mathbf{C}}\rangle$. It is straightforward to check that the linear order $\leq$ of $G$ extends to a linear linear order of $L$ by letting, for $x, y \in L$, $x \leq_{L} y$ iff $l k g+l k c \leq k l h+k l d$, where $x=g+c, y=h+d, g, h \in G$, $c, d \in \bar{C}, k, l \in \mathbb{Z}^{+}, k c, l d \in C$. Indeed,

- $\leq_{L}$ is well-defined.

If $x \in L$ then since $\mathbf{L}=\langle\mathbf{G}, \overline{\mathbf{C}}\rangle$, there exist $g \in G$ and $c \in \bar{C}$ such that $x=g+c$. Since $c \in \bar{C}$, there exists a positive integer $k$ such that $k c \in C$. Analogously, for $y \in L$, there exists $h \in G, d \in \bar{C}$, and a positive integer $l$ such that $y=h+d$ and $l d \in C$. Since $k c, l d \in C$ and $C \subseteq G$, it follows that $k c, l d \in G$. Therefore, referring to $g, h \in G$, it follows that $l k g+l k c, k l h+k l d \in G$, and hence it makes sense to compare them by $\leq$, the linear order of $G$.

Assume that it also holds that $x=g_{1}+c_{1}$ and $k_{1} c_{1} \in C$ and assume that it also holds that $y=h_{1}+d_{1}$ and $l_{1} d_{1} \in C$. Then, as above, $l_{1} k_{1} g_{1}+$ $l_{1} k_{1} c_{1}, l_{1} k_{1} h_{1}+l_{1} k_{1} d_{1} \in G$. We need to verify that $l k g+l k c \leq k l h+k l d$ if and only if $l_{1} k_{1} g_{1}+l_{1} k_{1} c_{1} \leq k_{1} l_{1} h_{1}+k_{1} l_{1} d_{1}$. If $l k g+l k c \leq k l h+k l d$ then $l k l_{1} k_{1}(g+c) \leq l k l_{1} k_{1}(h+d)$, then $l k l_{1} k_{1}\left(g_{1}+c_{1}\right) \leq l k l_{1} k_{1}\left(h_{1}+d_{1}\right)$. Since $l_{1} k_{1}\left(g_{1}+c_{1}\right), l_{1} k_{1}\left(h_{1}+d_{1}\right) \in G$, it follows that $l_{1} k_{1}\left(g_{1}+c_{1}\right) \leq l_{1} k_{1}\left(h_{1}+d_{1}\right)$. The other direction is analogous.

2 The multiplicative notation would make the notation of the proof of Lemma B.4 much more complicated, e.g. writing $\left(\left(g^{k}\right)^{n}\right)^{m}$ instead of $m n k g$. 
- $\leq_{L}$ extends $\leq$. If $x, y \in G$ then $x=x+0_{\bar{C}}, y=y+0_{\bar{C}}$, and $0_{C}=1 \cdot 0_{\bar{C}}$. Therefore, if $x \leq y$ then $1 \cdot 1 \cdot x+1 \cdot 1 \cdot 0_{C} \leq 1 \cdot 1 \cdot y+1 \cdot 1 \cdot 0_{C}$ and it is equivalent to $x \leq_{L} y$.

For the coming proof of reflexivity, antisymmetry, transitivity, and linearity of $\leq_{L}$ let $x, y, z \in L$ such that

$x=g+c, g \in G, c \in \bar{C}, k$ is a positive integer, and $k c \in C$,

$y=h+d, h \in G, d \in \bar{C}, l$ is a positive integer, and $l d \in C$,

$z=u+e, u \in G, e \in \bar{C}, m$ is a positive integer, and $m e \in C$.

- reflexivity of $\leq_{L}$ : if $x \in L$ then $k k g+k k c \leq k k g+k k c$ holds since $\leq$ is reflexive, yielding $x \leq_{L} x$.

- antisymmetry of $\leq_{L} \cdot x \leq_{L} y$ implies $l k g+l k c \leq l k h+l k d$ and $y \leq_{L} x$ implies $l k h+l k d \leq l k g+l k c$. Hence $l k g+l k c=l k h+l k d$, and thus $x=g+c=h+d=y$.

- transitivity of $\leq_{L}: x \leq_{L} y$ implies $l k g+l k c \leq l k h+l k d$ and thus

$$
l k m g+l k m c \leq l k m h+l k m d,
$$

whereas $y \leq_{L} z$ implies $l m h+l m d \leq l m u+l m e$ and thus

$$
l k m h+l k m d \leq l k m u+l k m e .
$$

By the transitivity of $\leq$ it follows that $l k m g+l k m c \leq l k m u+l k m e$, and since $k m g+k m c, k m u+k m e \in G$, it implies $k m g+k m c \leq k m u+k m e$ which is equivalent to $x \leq_{L} z$.

- linearity of $\leq_{L}$ : for $x, y \in L$, either $l k g+l k c \leq l k h+l k d$ or $l k h+l k d \leq$ $l k g+l k c$ must hold by the linearity of $\leq$. Therefore, $x \leq_{L}$ or $y \leq_{L} x$ holds, respectively.

Since $\leq_{L}$, the total order on $L$ extends $\leq$, the total order on $G$, in the rest of this proof we shall denote $\leq_{L}$ simply by $\leq$.

$\bar{C}$ is convex in $L$ : Let $x, y \in \bar{C}$ and $a \in L$ such that $x<a<y$. There exist $n, m \in \mathbb{Z}^{+}$such that $n x, m y \in C$, there exist $g \in G, d \in \bar{C}$ such that $a=g+d$, and there exists $k \in \mathbb{Z}^{+}$such that $k d \in C$. It follows that $C \ni k m n x-n m k d<n m k g<k n m y-n m k d \in C$ and $n m k g \in G$. Since $C$ is convex in $G$, it yields $n m k g \in C$ thus ensuring $g \in \bar{C}$ and hence $a=g+d \in \bar{C}$.

Since $\overline{\mathbf{C}}$ is a divisible subgroup of $\mathbf{L}$, and divisible subgroups are known to be direct summands [23], there exists $\mathbf{M} \leq \mathbf{L}$ such that $\mathbf{L}=\mathbf{M} \oplus \overline{\mathbf{C}}$. Both $M$ and $\bar{C}$, being subsets of $L$, are linearly ordered by the ordering of $L$, giving 
rise to consider the lexicographic ordering on $M \oplus \bar{C}$. It coincides with $\leq$. Indeed, let $x, y \in L$ with $x=a+c, y=b+d, a, b \in M, c, d \in \bar{C}$. Contrary to the statement assume that $x$ is larger than $y$ in the lexicographic order. Then either $a=b$ and $c>d$ which contradicts to $x \leq y$, or $a>b$. In the latter case $\bar{C} \ni d-c \geq a-b \geq 0 \in \bar{C}$ follows from $x \leq y$, yielding $a-b \in \bar{C}$ since $\bar{C}$ is convex in $L$. Therefore $a-b \in M \cap \bar{C}=\{0\}$, hence $a=b$, a contradiction.

Therefore, $\mathbf{L}=\mathbf{M} \overrightarrow{\times} \overline{\mathbf{C}}$. By the first isomorphism theorem for ordered

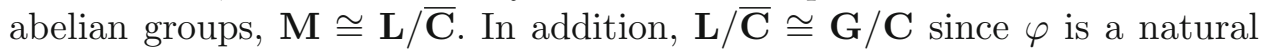
order isomorphism from $G / C$ to $L / \bar{C}$ given by $\varphi(g+C)=g+\bar{C}: \varphi$ is is welldefined and injective since $C \subseteq \bar{C}$ and since cosets of subgroups are known to be either disjoint or equal; $\varphi$ is surjective, too, since for $L \ni a=g+c$, $g \in G, c \in C$ it holds true that $\varphi(g+C)=g+\bar{C}=a-c+\bar{C}=a+\bar{C}$ since $C \subseteq \bar{C}$; and finally, $\varphi$ trivially preserves addition and the ordering. Summing up, $G \leq L=M \overrightarrow{\times} \bar{C} \cong G / C \overrightarrow{\times} \bar{C}$, that is, $\mathbf{G} \hookrightarrow \mathbf{G} / \mathbf{C} \overrightarrow{\times} \overline{\mathbf{C}}$. To see that the projection operation of $\mathbf{M} \overrightarrow{\times} \overline{\mathbf{C}}$ to the first coordinate maps $G$ onto $M$ let $a \in M$. Since $\mathbf{M} \leq \mathbf{L}=\langle\mathbf{G}, \overline{\mathbf{C}}\rangle, a=g+c$ for some $g \in G$ and $c \in \bar{C}$. Therefore, the unique decomposition of $g$ is $g=a-c$ and hence its projection to the first coordinate is $a$.

- Replace in item 4 of Lemma 6.2 the part "and (qua linearly ordered abelian groups) $\mathbf{X}_{\mathbf{g r}} \cong \beta\left(\mathbf{X}_{\mathbf{g}} \mathbf{r}\right) \overrightarrow{\times} \mathbf{k e r}_{\beta}$ " by "and (qua linearly ordered abelian groups) $\mathbf{X}_{\mathbf{g r}} \hookrightarrow_{\nu} \beta\left(\mathbf{X}_{\mathbf{g r}}\right) \overrightarrow{\times} \overline{\mathbf{k e r}_{\beta}}$." and replace the last 20 lines of the proof of Lemma 6.2 (starting with "By Hahn's theorem ...") by the sentence "Lemma B.4 ends the proof."

- Replace item 3 in Lemma 9.4 by

3. $\mathbf{X} \cong \mathbf{Y}_{\left(\mathbf{z} \overrightarrow{\times} \overline{\operatorname{ker}_{\beta}}\right)_{\mathrm{G}}}$ for some $\mathbf{G} \leq \mathbf{V} \overrightarrow{\times} \overline{\mathbf{k e r}_{\beta}}$.

- Replace the proof of item 3 in Lemma 9.4 by

3. Denote the embedding $\mathbf{X}_{\mathbf{g r}} \hookrightarrow_{\nu} \beta\left(\mathbf{X}_{\mathbf{g r}}\right) \overrightarrow{\times} \overline{\mathbf{k e r}_{\beta}}$ of Lemma 6.2 by $\delta=$ $\left(\left.\beta\right|_{X_{g r}}, \zeta\right)$. Define a mapping $\alpha: X \rightarrow Y_{Z_{V}} \overrightarrow{\times} \overline{k e r_{\beta}}$ by

$$
\alpha(x)=\left\{\begin{array}{ll}
(\gamma(\beta(x)), \zeta(x)) & \text { if } x \in X_{\tau<u} \\
(\gamma(\beta(x)), \top) & \text { if } x \in X_{\tau \geq u}^{T} \\
(\gamma(\beta(x)), \perp) & \text { if } x \in X_{\tau \geq u} \backslash X_{\tau \geq u}^{T}
\end{array} .\right.
$$

First we verify that $\alpha$ indeed maps into $Y_{Z_{V}} \overrightarrow{\times} \overline{k e r_{\beta}}$. By Definition 4.2 $Y_{Z_{V}} \overrightarrow{\times} \overline{k e r_{\beta}}$ is equal to $\left(V \times \overline{k e r_{\beta}}\right) \cup(Z \times\{\top\}) \cup(Y \times\{\perp\})$ which, by 
items 1 and 2 of Lemma 9.4, is equal to

$$
\left(\gamma\left(\beta\left(X_{\tau \geq u}^{E_{c}}\right)\right) \times \overline{k e r_{\beta}}\right) \cup\left(\gamma\left(\beta\left(X_{\tau \geq u}^{E}\right)\right) \times\{\top\}\right) \cup(\gamma(\beta(X)) \times\{\perp\}) .
$$

If $x \in X_{\tau<u}$ then $\gamma(\beta(x)) \stackrel{(9.1)}{=} \gamma\left(\beta\left(\top_{[x]}\right)\right)$ hence $\alpha(x) \stackrel{(B .2)}{=}(\gamma(\beta(x)), \zeta(x))$ is in $\gamma\left(\beta\left(X_{\tau \geq u}^{E_{c}}\right)\right) \times \overline{\operatorname{ker}_{\beta}}$. If $x \in X_{\tau \geq u}^{T}$ then $\alpha(x) \stackrel{(B .2)}{=}(\gamma(\beta(x)), \top) \in$ $\gamma\left(\beta\left(X_{\tau \geq u}^{E}\right)\right) \times\{\top\}$. If $x \in X_{\tau \geq u} \backslash X_{\tau \geq u}^{T} \subset X$ then $\alpha(x) \stackrel{(B .2)}{=}(\gamma(\beta(x)), \perp)$ is in $\gamma(\beta(X)) \times\{\perp\}$.

Using that $\gamma, \beta$, and $\zeta$ are homomorphisms (hence they preserve the order), $\zeta$ is strictly increasing (since $\delta$ is an embedding), and that $\top$ and $\perp$ are the top and bottom elements in the second coordinate, respectively, it is straightforward to verify that $\alpha$ is strictly increasing. Therefore, $\alpha$ preserves the ordering and $\alpha$ is injective. A straightforward verification using Definition 4.2/A and Table 3 shows that $\alpha$ preserves the monoidal operation, too. Indeed,

- if $x, y \in X_{\tau<u}$ then $x \otimes y \in X_{\tau<u}$ holds by Lemma 5.4. Adding that $\beta, \gamma, \zeta$ are homomorphisms, it follows that $\alpha(x \oplus y) \stackrel{(B .2)}{=}(\gamma(\beta(x \oplus$ $y)), \zeta(x \oplus y))=(\gamma(\beta(x)), \zeta(x)) \oplus_{\gamma}(\gamma(\beta(y)), \zeta(y)) \stackrel{(B .2)}{=} \alpha(x) \oplus_{\gamma} \alpha(y)$.

- if $x \in X_{\tau<u}$ and $y \in X_{\tau \geq u}^{T}$ then $x \circledast y \in X_{\tau \geq u}^{T}$ holds by Table $3_{(2,6)}$ and Table $3_{(2,3)}$. Adding the absorbing property of $T$, see Definition $4.2 / \mathrm{A}$, it follows that $\alpha(x \oplus y) \stackrel{(B .2)}{=}(\gamma(\beta(x \oplus y)), \top)=$ $(\gamma(\beta(x)), \zeta(x)) \otimes_{\gamma}(\gamma(\beta(y)), \top) \stackrel{(B .2)}{=} \alpha(x) \otimes_{\gamma} \alpha(y)$.

- if $x \in X_{\tau<u}$ and $y \in X_{\tau \geq u} \backslash X_{\tau \geq u}^{T}$ then $y \in X_{\tau \geq u} \backslash X_{\tau \geq u}^{T}$ holds by Table $3_{(2,4)}$. Adding the absorbing property of $\perp$, see Definition $4.2 / \mathrm{A}$, it follows that $\alpha(x \otimes y) \stackrel{(B .2)}{=}(\gamma(\beta(x \otimes y)), \perp)=$ $(\gamma(\beta(x)), \zeta(x)) \otimes_{\gamma}(\gamma(\beta(y)), \perp) \stackrel{(B .2)}{=} \alpha(x) \otimes_{\gamma} \alpha(y)$.

- if $x, y \in X_{\tau \geq u}^{T}$ then $x \circledast y \in X_{\tau \geq u}^{T}$ holds by Table $3{ }_{(3,3)}$, Table $3_{(3,6)}$ and Table $3_{(2,6)}$, hence, $\alpha(x \oplus y) \stackrel{(B .2)}{=}(\gamma(\beta(x \circledast y)), \top)=$ $(\gamma(\beta(x)), \top) \otimes_{\gamma}(\gamma(\beta(y)), \top)$

$$
\stackrel{(B .2)}{=} \alpha(x) \oplus_{\gamma} \alpha(y) \text {. }
$$

- if $x \in X_{\tau \geq u}^{T}$ and $y \in X_{\tau \geq u} \backslash X_{\tau \geq u}^{T}$ then $x \oplus y \in X_{\tau \geq u} \backslash X_{\tau \geq u}^{T}$ holds by Table $3_{(3,4)}$ and Table $3_{(6,4)}$. Adding the absorbing property of $\perp$, it follows that 


$$
\begin{aligned}
& \alpha(x \otimes y) \stackrel{(B .2)}{=}(\gamma(\beta(x \oplus y)), \perp)=(\gamma(\beta(x)), \top) \otimes_{\gamma}(\gamma(\beta(y)), \perp) \stackrel{(B .2)}{=} \\
& \alpha(x) \otimes_{\gamma} \alpha(y) .
\end{aligned}
$$

- if $x, y \in X_{\tau \geq u} \backslash X_{\tau \geq u}^{T}$ then $x \circledast y \in X_{\tau \geq u} \backslash X_{\tau \geq u}^{T}$ holds by Table $3_{(1,1)}$ and Table $3_{(4,4)}$. Adding the absorbing property of $\perp$, it follows that $\alpha(x \circledast y) \stackrel{(B .2)}{=}(\gamma(\beta(x \circledast y)), \perp)=(\gamma(\beta(x)), \perp) \otimes_{\gamma}(\gamma(\beta(y)), \perp) \stackrel{(B .2)}{=}$ $\alpha(x) \oplus_{\gamma} \alpha(y)$.

Moreover, (6.4), (7.3) and claim (4) in Proposition 8.2 shows the preservation of the residual complement, under $\alpha$. In complete analogy to the way we proved the preservation of the implication under $\beta$ in (6.5), we can verify the preservation of the implication under $\alpha$, too. Finally, it is clear that $\alpha$ maps the unit element of $\mathbf{X}$ to the unit element of $\mathbf{Y}_{\mathbf{Z}_{\mathbf{V}}} \overrightarrow{\times} \overline{\operatorname{ker}_{\beta}}$ : since $t \in X_{\tau<u}$, by (B.2) $\alpha(t)=(\gamma(\beta(t)), \zeta(t))$ holds and homomorphisms map unit elements to unit elements. Summing up, $\alpha$ is an injective homomorphism, that is, an embedding of $\mathbf{X}$ into $\mathbf{Y}_{\mathbf{Z}_{\mathbf{V}}} \overrightarrow{\times} \overline{\mathbf{k e r}_{\beta}}$. Therefore, to conclude the proof of item 3, it remains to prove that $\alpha$ is almost onto: if an element of $\mathbf{Y}_{\mathbf{Z}_{\mathbf{V}}} \overrightarrow{\times} \overline{\mathbf{k e r}_{\beta}}$ is not invertible then it is the $\alpha$-image of a suitable chosen $x \in X$. Indeed, since the product operation of $\mathbf{Y}_{\mathbf{Z}_{\mathbf{V}}} \overrightarrow{\times} \overline{\mathbf{k e r}_{\beta}}$ is defined coordinatewise by Definition 4.2, invertible elements of $\mathbf{Y}_{\mathbf{Z}_{\mathbf{V}}} \overrightarrow{\times} \overline{\mathbf{k e r}_{\beta}}$ are of the form $(a, b)$ where $(a, b) \in Y_{Z_{V}} \overrightarrow{\times} \overline{k^{2} r_{\beta}}$ and both $a$ and $b$ are invertible. By Definition 4.2 $Y_{Z_{V}} \overrightarrow{\times} \overline{k e r_{\beta}}$ is equal to

$$
\left(V \times \overline{k e r_{\beta}}\right) \cup(Z \times\{\top\}) \cup(Y \times\{\perp\}) .
$$

All elements of $V$ and $\overline{k e r_{\beta}}$ are invertible, since $V$ is a subgroup by Definition 4.2 and $\overline{k e r_{\beta}}$ is a convex hull of the subgroup ker $_{\beta}$ (see item 4 in Lemma 6.2). Therefore, if an element of $\mathbf{Y}_{\mathbf{Z}_{\mathbf{V}}} \overrightarrow{\times} \overline{\mathbf{k e r}_{\beta}}$ is not invertible then it is either of the form $(a, \top)$ where $a \in Z$, or the form $(a, \perp)$ where $a \in Y$. Consider $(a, \top)$ where $a \in Z$. By item 2 of Lemma 9.4, $Z=\gamma\left(\beta\left(X_{\tau \geq u}^{E}\right)\right)$. Therefore, $a=\gamma(\beta(x))$ for some $x \in X_{\tau \geq u}^{E}$. By letting

$$
y= \begin{cases}x & \text { if } x \in X_{\tau \geq u}^{T} \\ \top_{[v]} & \text { if } x=\perp_{[v]} \in X_{\tau \geq u}^{B}\end{cases}
$$

we obtain $\alpha(y)=\alpha(x) \stackrel{(B .2)}{=}(\gamma(\beta(x)), \top)=(a, \top)$ if $x \in X_{\tau \geq u}^{T}$ and $\alpha(y)=\alpha\left(\top_{[v]}\right) \stackrel{(B .2)}{=}\left(\gamma\left(\beta\left(\top_{[v]}\right)\right), \top\right) \stackrel{(9.1)}{=}\left(\gamma\left(\beta\left(\perp_{[v]}\right)\right), \top\right)=(\gamma(\beta(x))$, breaktop $)=(a, \top)$ if $x=\perp_{[v]} \in X_{\tau \geq u}^{B}$, and we are done. Consider $(a, \perp)$ 
where $a \in Y$. By item 1 of Lemma 9.4, $Y=\gamma(\beta(X))$, hence $a=\gamma(\beta(x))$ for some $x \in X$. By letting

$$
y= \begin{cases}\perp_{[x]} & \text { if } x \in X_{\tau<u} \\ x & \text { if } x \in X_{\tau \geq u} \backslash X_{\tau \geq u}^{T} \\ \perp_{[v]} & \text { if } x=\top_{[v]} \in X_{\tau \geq u}^{T_{c}} \\ x_{\downarrow} & \text { if } x \in X_{\tau \geq u}^{T_{p s}}\end{cases}
$$

we obtain $\alpha(y)=$

$$
\left\{\begin{aligned}
& \alpha\left(\perp_{[x]}\right) \stackrel{(B .2)}{=}\left(\gamma\left(\beta\left(\perp_{[x]}\right)\right), \perp\right) \stackrel{(9.1)}{=}(\gamma(\beta(x)), \perp)=(a, \perp) \\
& \text { if } x \in X_{\tau<u} \\
& \alpha(x) \stackrel{(B .2)}{=}(\gamma(\beta(x)), \perp)=(a, \perp) \\
& \text { if } x \in X_{\tau \geq u} \backslash X_{\tau \geq u}^{T} \\
& \alpha\left(\perp_{[v]}\right) \stackrel{(B .2)}{=}\left(\gamma\left(\beta\left(\perp_{[v]}\right)\right), \perp\right) \stackrel{(9.1)}{=}\left(\gamma\left(\beta\left(\top_{[v]}\right)\right), \perp\right)=(\gamma(\beta(x)), \perp)=(a, \perp) \text { if } x=\top_{[v]} \in X_{\tau \geq u}^{T_{c}} \\
& \alpha\left(x_{\downarrow}\right) \stackrel{(B .2)}{=}\left(\gamma\left(\beta\left(x_{\downarrow}\right)\right), \perp\right) \stackrel{(9.1)}{=}(\gamma(\beta(x)), \perp)=(a, \perp) \\
& \text { if } x \in X_{\tau \geq u}^{T_{p s}}
\end{aligned}\right.
$$

and we are done.

- Replace the second part of the last sentence before Proposition 10.1 (from "and finally we will recover ...") by the following.

and finally we will recover $\mathbf{X}$, up to isomorphism, as a type II partial sublex product of $\mathbf{X}_{\tau \geq u}, \mathbf{X}_{\tau \geq u}^{T}$, $\operatorname{ker}_{\beta}$ and a suitable chosen $\mathbf{G} \leq \mathbf{X}_{\tau \geq u}^{T_{c}} \overrightarrow{\times} \overline{\mathbf{k e r}_{\beta}}$.

- Replace item 3 in Lemma 10.2 by

3. $\mathbf{X} \cong\left(\mathbf{X}_{\tau \geq u}\right)_{\left(\mathbf{X}_{\tau \geq u}^{T} \overrightarrow{\times \mathbf{k e r}_{\beta}}\right)_{\mathbf{G}}}$ for some

$$
\mathbf{G} \leq \mathbf{X}_{\tau \geq u}^{T_{c}} \overrightarrow{\times} \overline{\operatorname{ker}_{\beta}} .
$$

- Replace the proof of item 3 of Lemma 10.2 by the following.

3. By the previous claim and by claim (3) in Proposition 10.1,

$$
\left(\mathbf{X}_{\tau \geq u}\right)_{\left(\mathbf{X}_{\tau \geq u}^{T_{c}}\right)} \overrightarrow{\times} \overline{\operatorname{ker}_{\beta}}
$$


is well-defined. Referring to Lemma 6.2 , let $\delta=\left(\left.\beta\right|_{X_{g r}}, \zeta\right)$ denote the embedding $\mathbf{X}_{\mathbf{g}} \mathbf{r} \hookrightarrow_{\nu} \beta\left(\mathbf{X}_{\mathbf{g r}}\right) \overrightarrow{\times} \overline{\mathbf{k e r}_{\beta}}$. Define a mapping $\alpha: X \rightarrow$ $Z_{V} \overrightarrow{\times} \overline{k e r_{\beta}}$ by

$$
\alpha(x)=\left\{\begin{array}{ll}
\left(\top_{\beta(x)}, \zeta(x)\right) & \text { if } x \in X_{\tau<u} \\
(x, u) & \text { if } x \in X_{\tau \geq u}
\end{array} .\right.
$$

$\alpha$ is clearly injective.

- Then, in the same proof, replace all (three) instances of $\operatorname{ker}_{\beta}$ by $\overline{\mathbf{k e r}_{\beta}}$, as detailed below:

Denote the monoidal operation of $\left(\mathbf{X}_{\tau \geq u}\right)_{\left(\mathbf{X}_{\tau \geq u}^{T_{c}}\right)} \overrightarrow{\times} \overline{\mathbf{k e r}_{\beta}}$ by $\diamond=(\circledast, \oplus)$.

Denote the residual complement of $\left(\mathbf{X}_{\tau \geq u}\right)_{\left(\mathbf{X}_{\tau \geq u}^{T_{c}}\right)} \overrightarrow{\times} \overline{\operatorname{ker}_{\beta}}$ by ${ }^{i}$.

Since $\alpha$ preserves multiplication and residual complements, and since both $\mathbf{X}$ and $\left(\mathbf{X}_{\tau \geq u}\right)_{\left(\mathbf{X}_{\tau \geq u}^{T_{c}}\right)} \overrightarrow{\times} \overline{\operatorname{ker}_{\beta}}$ are involutive, $\ldots$

- Replace the last sentence of the proof of Lemma 10.2 by the following.

Therefore, $\alpha$ is an embedding of $\mathbf{X}$ into $\left(\mathbf{X}_{\tau \geq u}\right)_{\left(\mathbf{X}_{\tau \geq u}^{T_{c}}\right)} \overrightarrow{\overrightarrow{\times}} \overline{\mathbf{k e r}_{\beta}}$, hence into $\left(\mathbf{X}_{\tau \geq u}\right)_{\left(\mathbf{X}_{\tau \geq u}^{T}\right)} \overrightarrow{\times} \overline{\operatorname{ker}_{\beta}}$. Summing up, $\mathbf{X} \cong\left(\left(\mathbf{X}_{\tau \geq u}\right)_{\left(\mathbf{X}_{\tau \geq u}^{T}\right)} \overrightarrow{\times} \overline{\mathbf{k e r}_{\beta}}\right)_{\mathbf{G}}$ for some $\mathbf{G} \leq \mathbf{X}_{\tau \geq u}^{T_{c}} \overrightarrow{\times} \overline{\operatorname{ker}_{\beta}}$.

- Replace Theorem 11.1 along with its proof by the following.

Theorem 11.1. If $\mathbf{X}$ is an odd involutive $\mathrm{FL}_{e}$-chain, which has only $n \in \mathbb{N}$, $n \geq 1$ positive idempotent elements then it has a partial sublex product group representation, that is, for $i=2, \ldots, n$ there exist totally ordered abelian groups $\mathbf{H}_{1}, \mathbf{H}_{i}, \mathbf{G}_{i}, \mathbf{Z}_{i-1}$ along with $\iota_{i} \in\{I, I I\}$ such that $\mathbf{X} \cong \mathbf{X}_{n}$, where for $i \in\{2, \ldots, n\}$,

$$
\mathbf{X}_{1}=\mathbf{H}_{1} \text { and } \mathbf{X}_{i}=\left\{\begin{array}{ll}
\mathbf{X}_{i-1}\left(\mathbf{z}_{i-1} \overrightarrow{\times} \mathbf{G}_{i}\right)_{\mathbf{H}_{i}} & \text { if } \iota_{i}=I \\
\mathbf{X}_{i-1}\left(\mathbf{X}_{i-1} \mathbf{g} \overrightarrow{\mathbf{r} \times \mathbf{G}_{i}}\right)_{\mathbf{H}_{i}} & \text { if } \iota_{i}=I I
\end{array} .\right.
$$

Notice that Theorem 11.1 claims isomorphism between $\mathbf{X}$ and $\mathbf{X}_{n}$ hence $\mathbf{X}_{n}$ and consequently for $i=n-1, \ldots, 2$, the $\mathbf{X}_{i}$ 's are claimed implicitly to exist (to be well-defined). By Definition B.3, using that $\left(\mathbf{X}_{i}\right)_{\mathbf{g r}}=\mathbf{H}_{i}$ holds for $i \in\{1, \ldots, n\}$, it is necessarily that

for $i=2, \ldots, n, \mathbf{Z}_{i-1} \leq \mathbf{H}_{i-1}, \mathbf{H}_{i} \leq \mathbf{Z}_{i-1} \overrightarrow{\times} \mathbf{G}_{i}$ and

for $i=2, \ldots, n$, if $\iota_{i}=I I$ then $\mathbf{H}_{i-1}$ is discretely embedded into $\mathbf{X}_{i-1}$. 
Proof. Let $\mathbf{X}=\left(X, \leq, \oplus, \rightarrow_{\circledast}, t, f\right)$. Induction by $n$, the number of idempotent elements in $X^{+}$. If $n=1$ then the only idempotent in $X^{+}$is $t$, hence Theorem 2.4 implies that $(X, \leq, \oplus, t)$ is a linearly ordered abelian group $\mathbf{H}_{1}$ and we are done. Assume that the theorem holds up to $k-1$ (for some $2 \leq k<n)$, and let $\mathbf{X}$ be an odd involutive $\mathrm{FL}_{e^{-c h a i n}}$ which has $k$ positive idempotent elements. Since the number of idempotents in $X^{+}$is finite, there exists $u$, the smallest idempotent above $t$.

If $u^{\prime}$ is idempotent then by Lemma 9.4 (by denoting $\alpha=\gamma \circ \beta$ )

$$
\mathbf{X} \cong \alpha(\mathbf{X})\left(\alpha\left(\mathbf{X}_{\tau \geq u}^{E}\right) \overrightarrow{\times} \overrightarrow{\operatorname{ker}_{\beta}}\right)_{\mathbf{H}_{i}}
$$

holds for some $\mathbf{H}_{i} \leq \alpha\left(\mathbf{X}_{\tau \geq u}^{E_{c}}\right) \overrightarrow{\times} \overline{\operatorname{ker}_{\beta}}$, where $\alpha\left(\mathbf{X}_{\tau \geq u}^{E_{c}}\right) \leq \alpha\left(\mathbf{X}_{\tau \geq u}^{E}\right)$ are subgroups of the odd involutive $\mathrm{FL}_{e}$-chain $\alpha(\mathbf{X})$, and $\mathbf{k e r}_{\beta}$ is a linearly ordered abelian group. Therefore, if $u^{\prime}$ is idempotent then set

$$
\mathbf{X}_{k-1}=\alpha(\mathbf{X}), \mathbf{Z}_{k-1}=\alpha\left(\mathbf{X}_{\tau \geq u}^{E}\right), \mathbf{G}_{k}=\overline{\operatorname{ker}_{\beta}} \text {, and } \iota_{k}=I .
$$

If $u^{\prime}$ is not idempotent then by Lemma 10.2

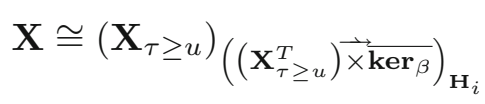

holds for some $\mathbf{H}_{i} \leq \mathbf{X}_{\tau>u}^{T_{c}} \overrightarrow{\times} \overline{\operatorname{ker}_{\beta}}$, where $\mathbf{X}_{\tau>u}^{T_{c}} \leq \mathbf{X}_{\tau>u}^{T}$ are subgroups of the odd involutive $\mathrm{FL}_{e}$-chain $\mathbf{X}_{\tau \geq u}$, and $\operatorname{ker}_{\beta}$ is a linearly ordered abelian group. Therefore, if $u^{\prime}$ is not idempotent then set

$$
\mathbf{X}_{k-1}=\mathbf{X}_{\tau \geq u}, \mathbf{G}_{k}=\overline{\operatorname{ker}_{\beta}} \text {, and } \iota_{k}=I I \text {. }
$$

By Lemmas 9.4 and 10.2 the number of positive idempotent elements of $\mathbf{X}_{k-1}$ (be it equal to either $\alpha(\mathbf{X})$ or $\mathbf{X}_{\tau \geq u}$ ) is one less than that of $\mathbf{X}$. Therefore, by the induction hypothesis, the theorem holds for $\mathbf{X}_{k-1}$, that is, there exist linearly ordered abelian groups $\mathbf{H}_{1}$ and for $i=2, \ldots, k-1$, linearly ordered abelian groups $\mathbf{H}_{i}, \mathbf{G}_{i}, \mathbf{Z}_{i-1}$ along with $\iota_{i} \in\{I, I I\}$ such that $\mathbf{X}_{1}:=\mathbf{H}_{1}$ and for $i \in\{2, \ldots, k-1\},(11.1)$ holds.

- In Remarks 11.2 and 11.3, in the paragraph before Corollary 11.5, in Corollary 11.5, and in the paragraph after Corollary 11.5 replace all instances of type III, type IV, and lexicographic by type I, type II, and sublex, respectively.

- In Remark 11.4, after "One can recover $\mathbf{X}$ as follows:" after the first three items insert a fourth item:

4. Replace the group-part of the obtained algebra by a subgroup of it. 
- Delete both instances of " $\mathbf{G}_{1} \ldots, \mathbf{G}_{n}$ " in the paragraph before Corollary 11.5.

- Replace Corollary 11.6 and its proof by

COROLLARY 11.6. The monoid reduct of any odd involutive $\mathrm{FL}_{e}$-chain which has only finitely many idempotent elements embeds in the finite lexicographic product $\mathbf{H}_{1} \overrightarrow{\times} \mathbf{G}_{2}^{\top \perp} \overrightarrow{\times} \ldots \overrightarrow{\times} \mathbf{G}_{n}^{\top \perp}$, where $\mathbf{H}_{1}, \mathbf{G}_{2}, \ldots, \mathbf{G}_{n}$ are the linearly ordered abelian groups of its group representation.

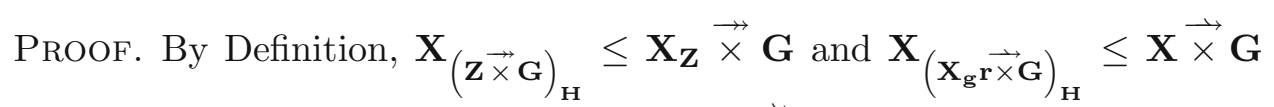
hold. Observe that the monoidal reduct of $\mathbf{X} \overrightarrow{\times} \mathbf{G}$ embeds into the monoidal

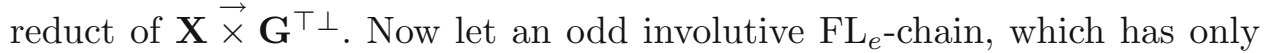
finitely many idempotent elements, be given. Take its group representation. Guided by its consecutive iterative steps, in each step consider $\left(\mathbf{X}_{i-1}\right) \overrightarrow{\times}$ $\mathbf{G}_{i}^{\top \perp}$ instead of $\mathbf{X}_{i-1}\left(\mathbf{z}_{i-1} \overrightarrow{\times} \mathbf{G}_{i}\right)_{\mathbf{H}_{i}}$ or $\mathbf{X}_{i-1}\left(\mathbf{X}_{i-1} \overrightarrow{\mathbf{g r}} \overrightarrow{\times} \mathbf{G}_{i}\right)_{\mathbf{H}_{i}}$.

In the end, this results in the original algebra being embedded into the lexicographic product $\mathbf{H}_{1} \overrightarrow{\times} \mathbf{G}_{2}^{\top \perp} \overrightarrow{\times} \ldots \overrightarrow{\times} \mathbf{G}_{n}^{\top \perp}$, where $\mathbf{H}_{1}, \mathbf{G}_{2}, \ldots, \mathbf{G}_{n}$ are linearly ordered abelian groups of its group representation.

- Finally, add [23], [24] and [25] to the references of [26].

Acknowledgements. The present scientific contribution was supported by the GINOP 2.3.2-15-2016-00022 grant and the Higher Education Institutional Excellence Programme 20765-3/2018/FEKUTSTRAT of the Ministry of Human Capacities in Hungary.

Publisher's Note Springer Nature remains neutral with regard to jurisdictional claims in published maps and institutional affiliations.

\section{References}

[23] BAER, R., Abelian groups that are direct summands of every containing abelian groups, Bull. Amer. Math. Soc. 46(10): 800-806, 1940.

[24] Fuchs, L., Infinite Abelian Groups, Vol. 1, Academic, New York, 1970; Mir, Moscow, 1974.

[25] Glass, A. M. W., Partially ordered groups, World Scientific, Singapore, 1999.

[26] Jenei, S., The Hahn embedding theorem for a class of residuated semigroups, Studia Logica 108(6): 1161-1206, 2020. 
Correction to: The Hahn Embedding Theorem...

S. JENEI

Institute of Mathematics and Informatics

University of Pécs

Ifjúság u. 6.

Pécs 7624

Hungary

jenei@ttk.pte.hu 